\title{
Gestión del inventario y el rendimiento financiero en las empresas automotrices, Tarapoto, 2020
}

\author{
Dr. César Augusto Flores Tananta \\ cflorest@ ucvvirtual.edu.pe \\ ORCID: 0000-0002-9336-1483 \\ Escuela de Administración \\ Universidad César Vallejo \\ Mtro. José Gabriel Arévalo Arévalo \\ jarevaloar@ucv.edu.pe \\ ORCID: 0000-0002-3344-7373 \\ Escuela de Administración \\ Universidad César Vallejo \\ MBA. Jhoanna Pérez Hidalgo \\ pperezhi90@ucvvirtual.edu.pe \\ ORCID: 0000-0002-8320-8573 \\ Escuela de Administración \\ Universidad César Vallejo

\section{Mtro. Julio Alberto Escalante Torres \\ jescalantet@ucv.edu.pe \\ ORCID: 0000-0001-9357-6807 \\ Escuela de Administración \\ Universidad César Vallejo}

\section{RESUMEN}

La gestión de inventarios, es el proceso de administración, de modo que se logre minimizar al máximo su cuantía, sin afectar la calidad del servicio al cliente, mediante un adecuado plan y control (Cespón Castro, 2012); asimismo Castillo (2012), indica que un adecuado registro de inventarios, significa contar con información suficiente y útil para disminuir los costes de producción e incrementar el rendimiento financiero de las empresas. Es por eso que la presente investigación se planteó como objetivo principal: Determinar la relación de la gestión del inventario y el rendimiento financiero en las empresas automotrices de Tarapoto, 2020, la población y la muestra fueron 30 empresas, el tipo de investigación fue aplicado; de enfoque cuantitativo, su diseño fue descriptivo 
correlacional, no experimental, de corte transversal, la técnica fue la encuesta y el instrumento el cuestionario; los autores concluyeron que, que la gestión de inventarios tiene una incidencia directa en el rendimiento financiero, tal como lo demuestran los resultados del coeficiente de Rho de Spearman con un 0.852 de correlación positiva alta y con una significancia del 0.963 con un gl. 28, siendo este un resultado menor al nivel de significancia del $\mathrm{p}$-valué $=0.05$ propuesto esperado ( $\mathrm{p}$-value $<0.05=\mathrm{Hi}$ ).

Palabras clave: gestión; inventarios; rentabilidad; estados financieros; ratios financieros 


\title{
Inventory management and financial performance in automotive companies, Tarapoto, 2020
}

\begin{abstract}
ABSTRAC
Inventory management is the administration process, so that its amount is minimized to the maximum, without affecting the quality of customer service, through an adequate plan and control (Cespón Castro, 2012); Likewise, Castillo (2012) indicates that an adequate inventory record means having sufficient and useful information to reduce production costs and increase the financial performance of companies. That is why the present investigation was raised as the main objective: To determine the relationship of inventory management and financial performance in the automotive companies of Tarapoto, 2020, the population and the sample were 30 companies, the type of investigation was applied; quantitative approach, its design was descriptive correlational, non-experimental, crosssectional, the technique was the survey and the instrument the questionnaire; The authors concluded that inventory management has a direct impact on financial performance, as shown by the results of the Spearman Rho coefficient with a 0.852 of high positive correlation and with a significance of 0.963 with a gl. 28, this being a result lower than the significance level of the proposed expected p-value $=0.05$ (p-value $<0.05=\mathrm{Hi})$.
\end{abstract}

Keywords: management; inventories; profitability; financial statements; financial ratios

Artículo recibido: 30 noviembre. 2021 Aceptado para publicación: 29 diciembre 2021 Correspondencia: cflorest@ucvvirtual.edu.pe Conflictos de Interés: Ninguna que declarar 


\section{INTRODUCCIÓN}

En los Estados Unidos-México, se pudo evidenciar, una de las principales razones por las que sufren los empresarios mexicanos, es que no contaban con controles efectivos de sus inventarios y de los saldos físicos en almacenes, esto les conducía a tomar decisiones equivocadas, debido a la falta de información clara en los informes o reportes de inventarios, lo cual afectó a las empresas mexicanas (Instituto Nacional de Estadística y Geografía - INEGI, 2019). Sin embargo “4 millones de deudas estaban relacionadas con la gestión de inventarios en la industria automotriz, expresadas en 1 millón 600 u.m, significa que las empresas cometieron 2.5 de delitos de alteración o malversación en la gestión de inventarios” (p.45). Así mismo, las pautas o procedimientos de control de inventarios prevalecieron ante cualquier intento de robo de mercaderías, suministros o dinero en efectivo (INEGI, 2019). Cabe precisar que " 5 de cada 5 pequeñas y medianas empresas, no han sobrevivido a más de 5 años en el mercado, sin una adecuada gestión de inventarios" (p.29).

Es preciso mencionar que, en el campo financiero, la gestión de inventarios representó y representa actualmente, una parte imprescindible del activo corriente en las pymes, siendo clasificado como un mecanismo importante que se encuentra en el rubro de activos corriente, los mismos que se pueden evidencia en el estado de situación financiera (Garrido \& Cejas, 2017). Es por ello que Suarez (2012), manifiesta que tener un inventario adecuado está relacionado al incremento de los márgenes de ganancias y al rendimiento financiero, anticipándose con ello el impacto de las políticas internas de las entidades de acuerdo a la cantidad de inventario; indagando en ese sentido reducir el costo total de las actividades de adquisición que aseguran el servicio de la compra que realiza un cliente.

Por otro lado, las deficiencias en las empresas del estado de México, han sido causados por la deficiente gestión de inventarios lo que trae como consecuencia que las ventas bajen, indistintamente de la existencia de otros factores que afectan a la administración de las empresas privadas. Además, el control de los productos físicos ha sido comparados con los reportes del sistema al finalizar el año fiscal debido a que se empleó mayor personal, lo que genera la contratación adicional por horas a pagar. Por lo tanto, estos inventarios pagados por horas representan el sobrecosto de trabajo, mientras dos empleados ejecutan correctamente las funciones de control del stock. 
En el Perú, el 75\% de industrias automotrices tienen deficiencias en la gestión del inventario, el cual, es uno de los principales motivos que genera el quiebre de las empresas pymes en este país; según Ofisis (2019), indica que las empresas en el campo de acción, las soluciones son los softwares de gestión empresarial; así lo evidenciaron las diferentes corporaciones del sector industrial, construcción, automotriz, energía y minería. Si bien es cierto, la implementación del software puede ser importante, pero no imprescindible, porque el sistema puede agilizar el procedimiento del control de las mercaderías en un $45 \%$, además. Sin embargo. "La gestión de inventario tradicional o no automatizada, solo reduce en un $25 \%$ a $30 \%$ los procesos de inventariados" (p.77).

En la provincia de San Martín, se evaluó a las empresas dedicadas al rubro comercial de servicios automotrices ubicada en la intemperie del distrito de Tarapoto, cabe indicar que los servicios que ofrecen al público en general son a precios accesibles. Entre los servicios que brindan a sus clientes se observan el enllante, desenllante, balanceo de motores, escaneo computarizado, mantenimiento preventivo, reparación parcial y general, revisión de sistema eléctrico, etc. También ofrecen la venta de repuestos de todo tipo de vehículos, batería, filtros de la marca ETNA. De las 30 empresas tomados como muestra, se optó por conversar con los trabajadores que se encuentran en la gerencia general, en el área de ventas, taller y logística. Sin embargo, la mayoría de las empresas no cuentan con un sistema automatizado sobre la gestión de las existencias, solo cuenta con control manual, lo que siempre genera descuadre e inconsistencia en los saldos y esto arrastra muchos errores en los reportes del del control físico de los suministros, repuestos, accesorios, lubricantes y otros bienes que las empresas comercializan.

Aunado a esto, los profesionales que están a cargo de la gestión de los inventarios, son personas sin mucha experiencia en el rubro y en manejos de sistemas y etas falencias son por falta de decisión de la gerencia. Cabe indicar que los inventarios generalmente desarrollan un rol muy importante en la funcionalidad de toda organización, "que está dedicada a la compra o venta de bienes o servicios, de aquí la importancia del manejo correcto de estos por parte de la misma, para obtener el mejor rendimiento financiero" (Douglas Thomas, 2000, p.620). Si las empresas no cambian sus sistemas desfasados y tampoco les interesa conocer de manera concreta que están teniendo fugas de dinero por causa de un sistema que solo retrasa las actividades y confunde las cantidades que se colocan en los requerimientos para ejecutar las adquisiciones diversas, estas acciones, 
lejos de permitir optimizar los costos, solo mostraron inconsistencias e incongruencias en los saldos de inventarios y esto afectan la parte económica y financiera de las organizaciones; específicamente la rentabilidad.

Por otro lado, la cantidad económica de los pedidos, busca conseguir la optimización del costo total, mediante la determinación de órdenes de compra para el reabastecimiento, esto con la finalidad de reducir los pedidos de compras. El propósito de calcular Economic Order Quantity - EOQ (Cantidad Económica de Pedido), es reducir la combinación de los precios, tales como los costos de las compras de los inventarios, costos de almacenamientos y costos de pedidos (Ladrón De Guevara, 2020).

Sin embargo, Winston Wayne (2004), indica que el propósito se centra en determinar las reglas que puede usar la gerencia para reducir los costos asociados con mantener el inventario y crear la oportunidad de satisfacer las exigencias de los clientes.

Además, las empresas buscan gestionar el inventario, mediante la creencia del Just in Time (justo a tiempo), esta mecánica ha permitido generar grandes ahorros mediante la optimización de los inventarios al mínimo (Hillier y Lieberman, 2010).

Asimismo, consideran que "Las existencias son materiales y accesorios que se esperan usar o utilizar en el proceso de elaboración, para el investigador es importante determinar un nivel óptimo y una rotación de las existencias, que permita el crecimiento de la organización" (Vizcarra Moscoso, 2007, p.200).

Es por eso que Moreira et al. (2019), nos menciona que el enfoque de las existencias, "se basa en que la entidad administre los suministros requeridos de acuerdo a la cantidad solicitada por el cliente y esto origina la eficiencia del costo del inventario, mejor calidad de atención y servicio" (p.11).

Por eso, Meana Coalla (2013), describe en su libro a la gestión de inventarios, como la verificación y control de las existencias o activos de la empresa como ganancia o pérdida. Así mismo Bravo Carrasco (2009), opina que la gestión del inventario, es el punto más importante de la entidad, debido a que en la rotación se centran las ganancias, porque es fundamental para la gestión estratégica.

La variable de la rentabilidad, por las causas que se describen líneas arriba, durante los últimos dos años (2019 y 2020), se ha visto afectada en un $37 \%$ sobre el volumen de ventas que las empresas han proyectado anualmente, ese porcentaje representa en términos cuánticos un total de S/ 1971406.25 soles, los cuales se han venido arrastrando 
durante todo ese tiempo; tal es el caso del año 2019, donde se observó una rentabilidad del $10 \%$ menor a lo esperado, el mismo que equivale a S/ 532812.50 soles, en el año 2020 se identificó una rentabilidad del 15\% inferior a lo que se había proyectado, el cual equivale a un total de S/ 799218.75 soles; y en estos 8 primeros meses del año, el rendimiento financiero fue del $12 \%$ menor a lo planificado, lo que significa un total de $\mathrm{S} /$ 639375.00 soles. Esto resulta muy alarmante, desde la perspectiva de la sostenibilidad, debido a que, si esta esta problemática no se soluciona, las pérdidas pueden ser más cuantiosas, lo que podría generar pérdidas irremediables, el mismo que llevaría a quebrar a algunas empresas.

Ante esto Muller y De Camino (2014), define la rentabilidad como una. "Expresión económica resultante de la diferencia entre ingresos y costos, el cual está sujeto a restricciones relacionadas con el medio ambiente o la sociedad" (p.238).

Del mismo modo, Lizcano y Castello (2004), la rentabilidad. "Son los recursos financieros que se logran por una buena gestión de la directiva" (p.120).

Por otro lado, Alberca y Rodríguez, (2012), conceptualizan la rentabilidad, como el redito económico obtenido por la compañía, productos de las diversas transacciones económicas dentro de un período fiscal.

De similar forma Apaza Meza (2010), afirma que la rentabilidad se concentra en la capacidad de generar réditos económicos, Además se considera uno de los indicadores más relevantes para calcular el éxito de una corporación.

En efecto Adrianzen Cabrera (2016), afirman que la rentabilidad. "Es la relación entre los beneficios y los recursos utilizados para tal fin" (p.125).

De similar forma Coello Portugal (2017), considera que la rentabilidad. "Permite conocer el grado de utilidad que proviene del eficiente uso del dinero invertido en las compañías" (p.180).

Además, Ferrer Cárdenas (2014), piensa que, para entender eficazmente la gestión financiera de una entidad. "Es fundamental tomar en cuenta el rendimiento financiero o rentabilidad, porque estos indicadores son los que permitieron identificar el capital de la empresa" (p.188).

Escribano y Jiménez (2014), piensan que el análisis de rentabilidad. "Es una información de carácter relevante y de suma importancia en una empresa, porque muestra las ganancias y pérdidas que la empresa ha obtenido en un período contable” (p.431). 
Por otro lado, se piensa que la rentabilidad son los beneficios de una transacción económico-financiera y su relación con la inversión en proyectos de temas financieros (Sánchez y Jaramillo, 2016).

Pero Román López (2012), afirma que la rentabilidad, es uno de los indicadores financieros más importantes porque determina el éxito o el fracaso de una empresa.

Mientras que Guzmán Álvarez (2010), piensa que la razón es un conjunto de índices de la relación entre las dos cuentas, es decir, la tabla de estado financiero y la tabla de ingresos.

$\mathrm{Al}$ respecto Mafra, et al. (2016), la rentabilidad muestra el desempeño financiero que la empresa cree pertinente para la toma de decisiones sobre financiamiento.

Así mismo, Hernández \& Ríos (2013), indica que es una variable específica, ya que mide la estructura financiera, la combinación de la deuda y el capital destinado al financiamiento, cabe precisar qué para medir el rendimiento de una entidad, se debe controlar costos o gastos y convertir ventas en ganancias, se usan los indicadores de rentabilidad.

De esto se desprende que los índices más utilizados para medir el rendimiento financiero - rentabilidad de acuerdo a Goldman (2017) son el retorno sobre las ventas (ROS), retorno sobre los activos (ROA) y retorno sobre el patrimonio (ROE), para propósito de este artículo se usó el ROA y ROE, por ser más específicos.

Finalmente, una de las actividades que más rentabilidad ha producido dentro de las empresas, han sido todos los suministros que se utilizan como repuesto de los vehículos que entran a mantenimiento y reparación, pero es importante mencionar que este de tipo de mercaderías representó uno de los mayores problemas, debido a que ocasiona muchas dificultades para registrar o ingresar el descargo en el sistema del software SICO, que dicho sea de paso; está obsoleto, lo que generó incongruencias con los saldos que emiten los responsables del control de las unidades físicas. Cabe indicar que los servicios de venta de suministros varios, pasan por un proceso de control de salida de almacén, dichos bienes carecen de una correcta organización, zonificación, ubicación, rotulado, entre otros. Y en ciertos casos los productos son colocados en el piso.

Estas deficiencias descritas con antelación, dificultaron la recepción y solicitud de pedidos que la empresa debe ejecutar de manera diaria; Además, ciertas estanterías no se encuentran señalizadas generando problemas para colocar los bienes en un lugar 
adecuado y al buscar los productos para vender o utilizar en los servicios que presta la empresa; No se pueden ubicar o se encuentran con dificultad.

Por todo lo expuesto se ha planteado como objetivo general: Determinar la influencia de la gestión del inventario en el incremento de la rentabilidad de las empresas automotrices. Tarapoto 2020; así mismo se planteó los siguientes objetivos específicos: i) Conocer la gestión de inventarios en las empresas automotrices, Tarapoto 2020; ii) Calcular el nivel de rentabilidad de las empresas automotrices, Tarapoto 2020; iii) Describir cuál de las dimensiones de la gestión del inventario influye en la rentabilidad de las empresas automotrices, Tarapoto 2020.

\section{ESTRATEGIAS METODOLÓGICAS O MATERIALES Y MÉTODOS}

La metodología aplicada en este estudio de investigación fue de tipo aplicado, de diseño no experimental de corte transversal, de nivel descriptivo correlacional, con enfoque cuantitativo, así mismo se utilizó la lógica y el criterio deductivo, el cual consiste en la utilización de los principios teóricos y normativas vigentes sobre la gestión del inventario y el rendimiento financiero en las empresas automotrices, Tarapoto, 2020, la población y la muestra estuvo compuesta por 30 empresas automotrices de la provincia de San Martín, la muestra utilizada para la siguiente investigación fue de 30 profesionales que pertenecen a las empresas, tomando en cuenta específicamente de las áreas de: Mantenimiento, ventas, abastecimiento y administración, los cuales son contratados bajo el régimen laboral de la Ley $N^{\circ} 728$ - Ley de promoción y competitividad laboral; Por otro lado se analizó todos los datos que recolectamos utilizando la técnica de la encuesta y el instrumento del cuestionario, con la finalidad de conocer y comprender la eficiencia y eficacia de las dimensiones planteadas en la variable de Gestión de inventarios: Verificación de inventarios y control de inventarios; en cuanto a la variable de la Rendimiento financiero se consideró las dimensiones de: Rentabilidad sobre el patrimonio - ROE, la rentabilidad sobre los activos - ROA.

Sin embargo, toda investigación científica debe demostrar confiabilidad y seguridad de los instrumentos de aplicación de recolección de datos; Es por eso que, Alarcón R. (1991), define a la confiabilidad y seguridad de una herramienta, como el equilibrio y estancia de los resultados esperando que no evidencien cambios significativos en el transcurso de una serie de estudios aplicando el test.

Por otro lado, Córdova, I. (2013), manifiesta que un instrumento es confiable y seguro, 
cuando tiene la habilidad de realizar cálculos de una variable en distintas etapas cercanas. La técnica referenciada más usada y más confiable por los autores, citados con antelación para calcular la seguridad de la herramienta es el; Alpha de Cronbach, el mismo que se aplicó como piloto a 8 colaboradores de la empresa; cabe precisas que la validez del instrumento se hizo mediante el juicio de 3 expertos.

Es concordancia con las exigencias del artículo científico, es preciso mencionar que para la validación del instrumento de recolección de datos su utilizo el juicio de expertos, el cual es un método de validación útil para verificar la fiabilidad de una investigación que se define como: "Una opinión informada de personas con trayectoria en el tema, que son reconocidas por otros como expertos cualificados en éste, y que pueden dar información, evidencia, juicios y valoraciones” (Escobar-Pérez y Cuervo-Martínez, 2008:29).

\section{RESULTADOS Y DISCUSIÓN}

De acuerdo a la tabulación y procesamiento de la información recopilada sobre las variables de: Gestión de inventarios y rendimiento financiero en las empresas automotrices. Se obtuvieron los siguientes resultados sobre el análisis de las siguientes dimensiones que se describen a continuación.

\section{Objetivo específico 1:}

- Conocer la gestión de inventarios en las empresas automotrices, Tarapoto 2020.

\section{Figura 1}

Dimensión de la verificación de inventarios de la variable de gestión de inventarios

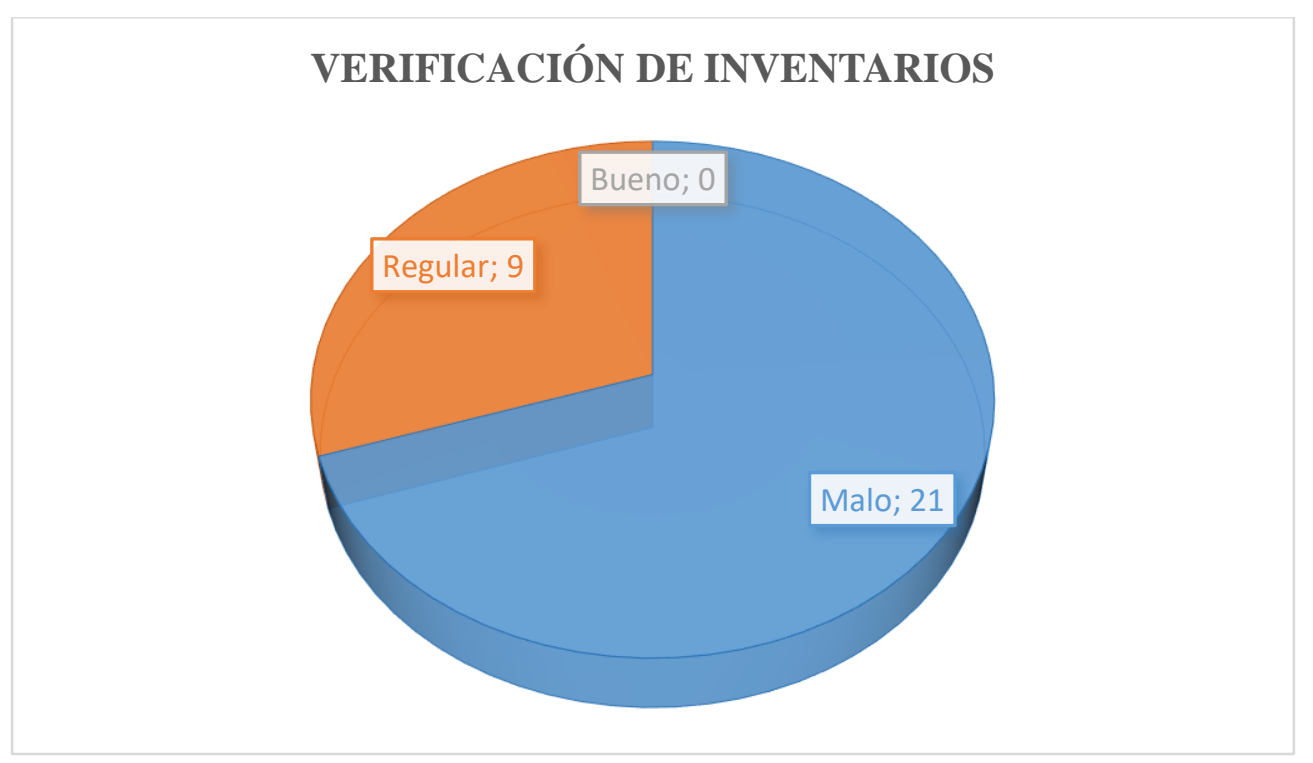

Fuente: Elaboración propia (Excel) 


\section{Interpretación}

En la figura 1; se observa que las empresas automotrices, después de la aplicación del instrumento de recopilación de datos a los 30 trabajadores; el 83\% de los encuestados consideró que la gestión del inventario desde el punto de vista de la verificación, es malo y el $17 \%$ piensa que es regular; esto significa que la empresa no verifica sus inventarios de manera permanente, eficiente y eficaz, lo que afecto la gestión de inventarios y por ende el rendimiento financiero que muy bien la empresa podría utilizar para realizar inversiones en el corto plazo, con la intención de hacer que las empresas sean más rentables y solventes.

\section{Figura 2}

Dimensión de control de inventarios de la variable de gestión de inventarios

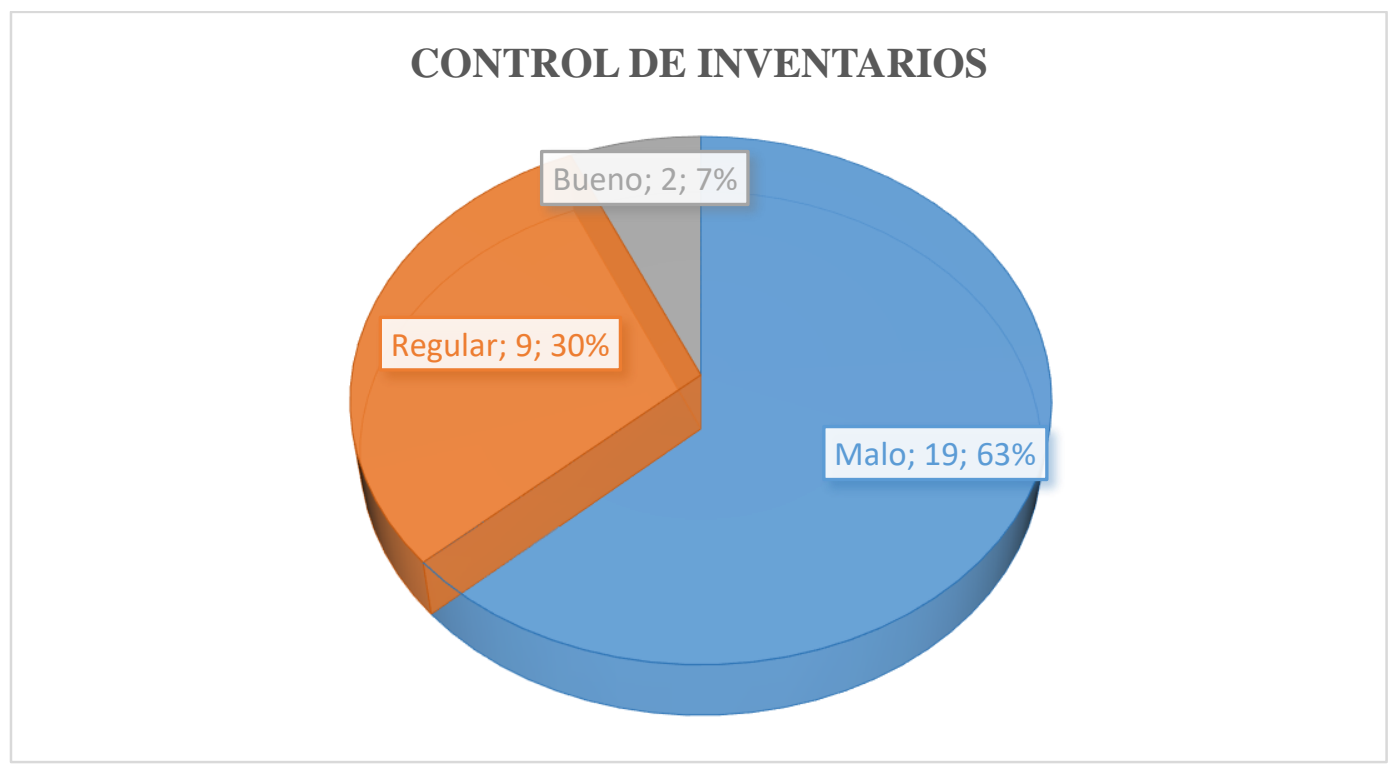

Fuente: Elaboración propia (Excel)

\section{Interpretación}

En la figura 2; se observa que las empresas automotrices, después de la aplicación del instrumento de recopilación de datos de 30 trabajadores; el 563\% de los encuestados opinó que el control de los inventarios, es malo; el 30\% consideró que el control de los inventarios es regular y el 7\% pensó que es bueno. Estos resultados dieron a conocer que el control de inventarios que se realizan en las empresas es ineficiente e inadecuado, el mismo que conlleva a pérdidas cuantiosas de dinero, afectando al disponible de las empresas . 


\section{Figura 3}

Consolidado del objetivo específico $N 1$

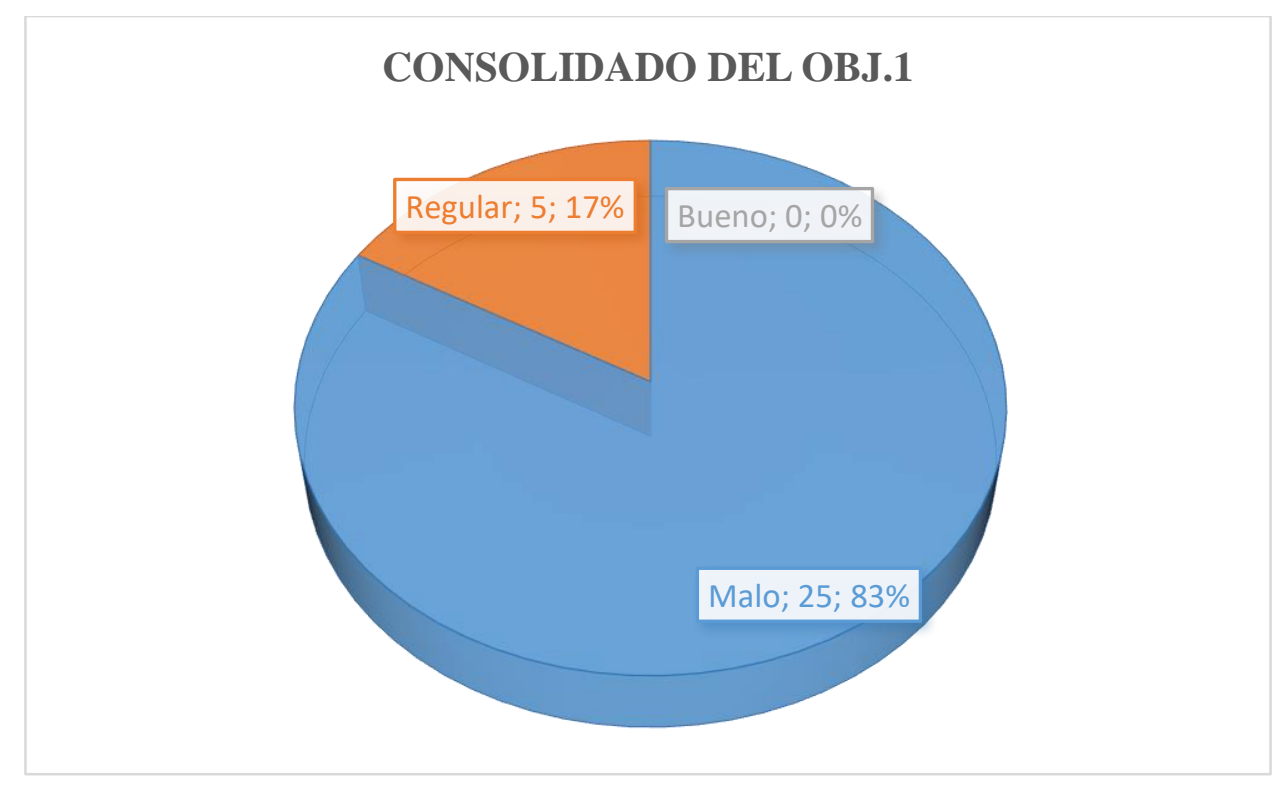

Fuente: Elaboración propia (Excel)

\section{Interpretación}

En la figura 3; se observa que las empresas automotrices, después de la aplicación del instrumento de recopilación de datos de 30 trabajadores; el $83 \%$ de los encuestados opinó que la gestión del inventario que la empresa realiza, es mala y el 17\% consideró que la gestión del inventario en regular. Lo que significó que hubo una opinión divergente, entre los involucrados, pero claramente se observó que la gestión de inventarios es inadecuada lo que debe preocupar a las gerencias de las diferentes empresas del medio dedicado al rubo de servicios automotrices.

En concordancia con esto Golaś Zbigniew (2020), concluyo que existe una disminución en la participación de los inventarios del activo total como parte de una deficiente gestión de inventario, evidenciándose claramente una reducción de manera diaria en el rubro de repuestos y otros materiales terminados, Así mismo Vásconez et al. (2020) indico que es sugerido concientizar y generalizar esta imprescindible herramienta de gestión de inventarios ya que responde a las necesidades. 


\section{Objetivo específico 2:}

- Calcular el nivel de la rentabilidad perdida de las empresas automotrices.

\section{Figura 4}

Dimensión del ROA de la variable de rendimiento financiero

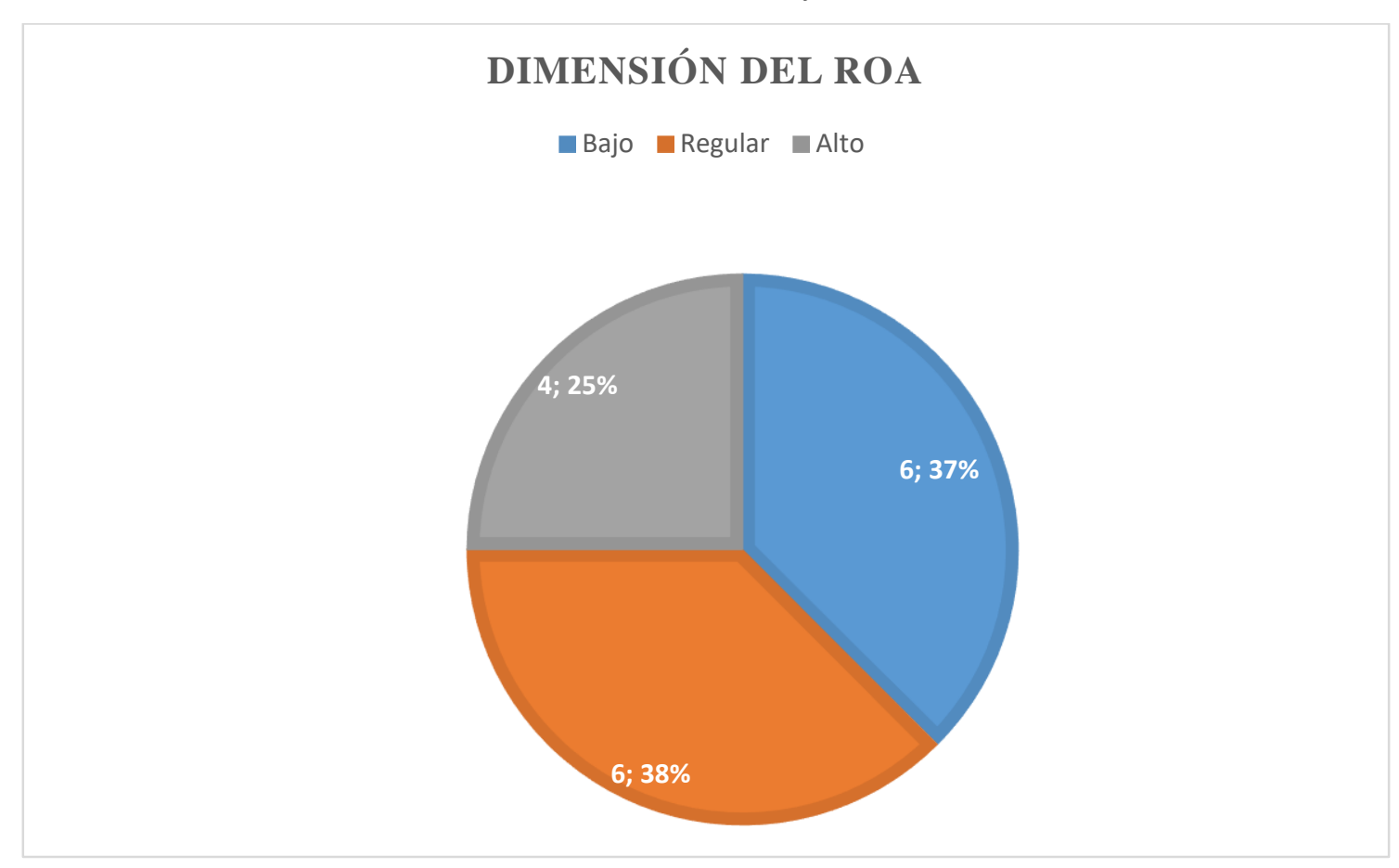

Fuente: Elaboración propia

\section{Interpretación}

En esta figura 4; se visualiza que las empresas automotrices, después de la aplicación del instrumento de recolección de datos, los trabajadores del área de finanzas de las empresas automotrices, han opinado que el $37 \%$ de ellos tienen un ROA bajo, mientras que el $38 \%$ piensa que el ROA, es Regular y solo el 25\% considera que el ROA que tienen las empresas es alto; éstos resultados evidencian que la gestión por inventarios repercute en el rendimiento financiero del activo sobre las generación de ingresos, por lo que se insta que se tomen medidas urgentes en cuanto a la mejora de los procesos de control y verificación de inventarios. 
Figura 5 Dimensión del ROE de la variable de rendimiento financiero

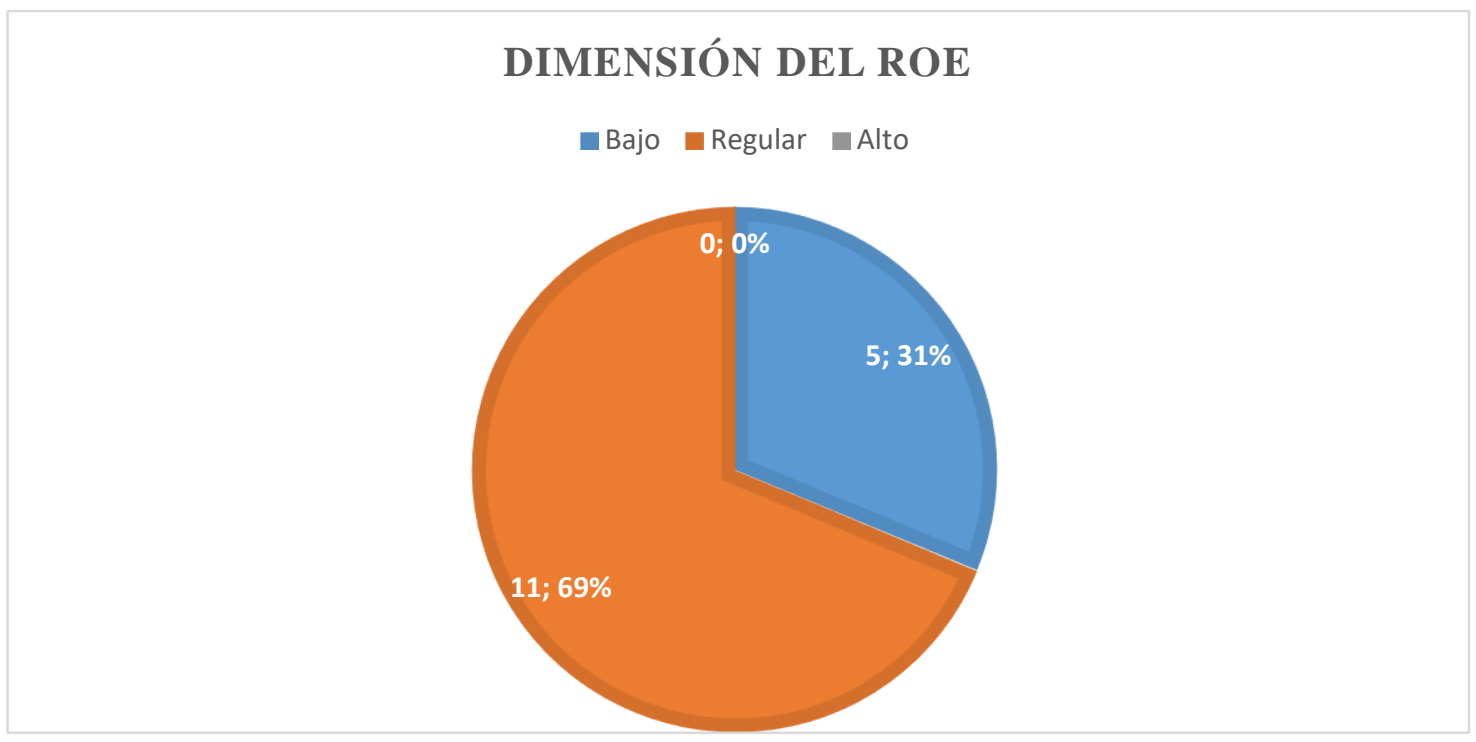

Fuente: Elaboración propia

\section{Interpretación}

En esta figura 5; se visualiza que las empresas automotrices, después de la aplicación del instrumento de recolección de datos, los trabajadores del área de finanzas de las empresas automotrices, han opinado que el $31 \%$ de ellos tienen un ROE bajo, mientras que el $69 \%$ piensa que el ROE, es Regular; éstos resultados evidencian que la gestión por inventarios repercute en el rendimiento financiero del patrimonio sobre las generación de utilidad neta, es por esto que se ha visto necesario capacitar más a los trabajadores encargados de la gestión de inventarios, con la finalidad de que no afecte el ROE.

Figura 6 Consolidad de la variable de rendimiento financiero

\section{VARIABLE DE RENDIMIENTO FINANCIERO}

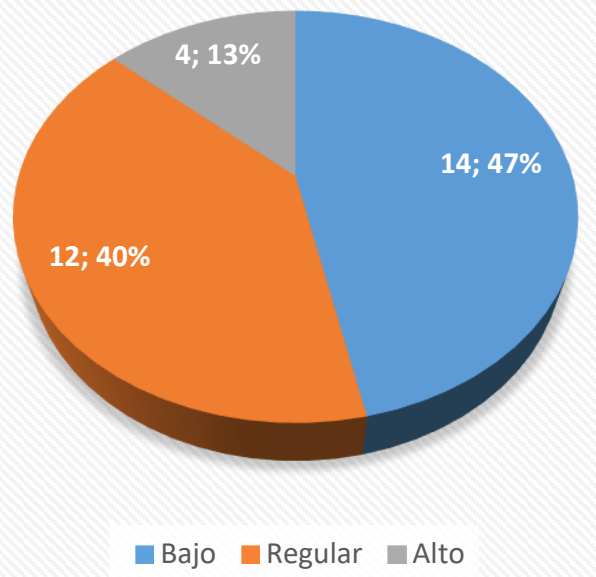

Fuente: Elaboración propia (EE. FF 2020 Y 2019) 


\section{Interpretación}

Tal como se observa en la figura 6; se visualiza que las empresas automotrices, después de la aplicación del instrumento de recolección de datos, los trabajadores del área de finanzas de las empresas automotrices, han opinado que el $47 \%$ de ellos tienen un rendimiento financiero bajo, mientras que el $40 \%$ piensa que es Regular y solo el $13 \%$ de estos profesionales piensan que es bueno; estos resultados evidencian que la rentabilidad en las empresas no es eficiente ni adecuado.

\section{Objetivo específico 3:}

- Describir en qué medida la gestión de inventarios influye en el rendimiento financiero en las empresas automotrices, Tarapoto 2020

Tal como se observó en las figuras antes descritas, se evidencia que la gestión del inventario es ineficiente e inadecuado; porque no muestra una correcta verificación de los productos que son adquiridos por la empresa y esto se contrastó con las respuestas de los trabajadores encuestados, que manifestaron que hay mala planificación, procedimientos inadecuados, Kardex manejado por profesionales que desconocen el proceso y sugerencias de auditorías que no se cumplen, porque los dueños aducen que son muy costosas implementarlas.

No obstante, el control de inventarios en la empresa, no se ha venido realizando de manera correcta, porque el método de inventario adoptado o ejecutado, no es actualizado permanentemente, el sistema de control de inventarios es deficiente, así mismo el control patrimonial es un desorden lo que dificulta y quita tiempo en los trabajos de mantenimiento de los vehículos.

Sin embargo, este mal accionar y falta de decisión en la mejora de la gestión del inventario de los socios fundadores, afectó significativamente la rentabilidad de las empresas, tal como lo demostraron los resultados de los indicadores financieros del ROA Y ROE.

\section{Objetivo general:}

- Determinar la influencia de la gestión de inventario en el incremento del rendimiento financiero en las empresas automotrices, Tarapoto 2020

\section{Tabla 1}

Correlación entre la gestión de inventarios y el rendimiento financiero.

Gestión de Rendimiento




\begin{tabular}{|c|c|c|c|c|}
\hline & & & inventarios & financiero \\
\hline & Gestión de & Coeficiente de & 1,000 & ,852 \\
\hline & Inventarios & correlación & & \\
\hline & & Sig. (bilateral) & . & ,963 \\
\hline Rho de & & $\mathrm{N}$ & 30 & 14 \\
\hline Spearman & Rendimient & Coeficiente & 852 & 1,000 \\
\hline & o financiero & correlación & & \\
\hline & & Sig. (bilateral) & ,963 & . \\
\hline & & $\mathrm{N}$ & 30 & 14 \\
\hline
\end{tabular}

Fuente: Statistical Package for the Social Sciences-spss v.26

\section{Interpretación}

En el detalle de la tabla 1, se puede apreciar que la significancia bilateral de los resultados fue menor que el nivel de "p.valúe" valor propuesto $(\mathrm{p}=0.963<0.05)$, este resultado permitió tomar la decisión de rechazar la hipótesis Nula o negativa (Ho) y aceptar la hipótesis positiva o alterna (Hi), Así mismo, el Rho Spearman que se determinó fue un valor de $(\mathrm{Rho}=0,852)$, contrastándose una correlación positiva - alta, es decir, se puede afirmar que existe una relación directa entre la gestión de inventarios y el rendimiento financiero en las empresas automotrices de Tarapoto, 2020.

Por otro lado, Sekeroglu \& Altan (2017), esta de acuerdo cuando se afirma que existe una relación positiva entre la gestión de los inventarios y la rentabilidad de la industria de comestibles. Asimismo, se identificó que existe una fuerte significancia entre las dimensiones de la gestión de inventarios y la rentabilidad en las empresas.

De similar forma Ülker et al. (2020), la rotación de las existencias, tiene una relación fuerte y significativa con la tasa de rendimiento de los activos, el capital de trabajo bruto y la tasa de rendimiento de capital social. Arenas \& Lindoro (2020), también demostraron que la gestión de costos de los inventarios y la rentabilidad de los restaurantes se encuentran relacionados entre sí, de acuerdo al Chi cuadrado calculado, con una significancia menor al 0.05; esperado, finalmente Ávila \& López (2019), manifiestan que los factores principales que intervienen en la decisión de incorporar un eficiente control interno de inventario basado en el modelo COSO ERM, tienen influencia significante en la rentabilidad de las empresas textiles el tejido en el emporio comercial de Gamarra.

\section{CONCLUSIÓN O CONSIDERACIONES FINALES}


- Los resultados encontrados evidenciaron que la gestión de inventarios tiene una incidencia directa en el rendimiento financiero, tal como lo demuestran los resultados del coeficiente de Rho de Spearman con un 0.852 de correlación positiva alta y con una significancia del 0.963 , con un grado de libertad $(\mathrm{gl}=28)$ siendo este un resultado menor al nivel de significancia del $\mathrm{p}$-valué $=0.05$ propuesto esperado ( $\mathrm{p}$-value $<0.05$ $=\mathrm{Hi})$.

- El 77\% de los trabajadores opinaron que la gestión del inventario que la empresa realiza, es deficiente, por lo que perjudica considerablemente en la economía de las empresas y en la eficiencia del servicio que se vende.

- El rendimiento financiero, en las empresas es regular de acuerdo a lo que indican los trabajadores especialistas en el tema, el cual evidencia la pésima gestión de inventarios que se ha realizado en esos dos últimos períodos contables. Por defecto esto afectó directamente a la utilidad neta de ambos períodos. Esta situación preocupa a los socios de la empresa.

- La gestión del inventario es ineficiente e inadecuado; porque no muestra una correcta verificación de los productos que son adquiridos por la empresa y esto se contrastó con las respuestas de los trabajadores encuestados, que manifestaron que hay mala planificación, procedimientos inadecuados, Kardex manejado por profesionales que desconocen el proceso y sugerencias de auditorías que no se cumplen, porque los dueños aducen que son muy costosas implementarlas.

\section{LISTA DE REFERENCIAS}

Arenas, F. G., \& Lindoro, G. E. (2020). El impacto de la gestión de costos de los inventarios en la rentabilidad de los restaurantes de comida criolla en el distrito de Surquillo durante el año 2018. Universidad Peruana de Ciencias Aplicadas (UPC). https://repositorioacademico.upc.edu.pe/handle/10757/652520

Ávila Hurtado, Y., \& López Duran, F. (2019). Control Interno de Inventarios basado en el modelo COSO ERM y su impacto en la Rentabilidad de las Empresas MYPES de Fabricación de Telas de Tejido Punto en el Emporio Comercial de Gamarra, año 2018. Universidad Peruana de Ciencias Aplicadas (UPC). https://doi.org/10.19083/tesis/651640

Alarcón R. (1991), en su libro de Métodos y diseños de investigación del comportamiento. Vol. 12. $\mathrm{N}^{\circ} 1$ 
APA (2016), Normas de la American Psychological Association - APA. Association 7th. Ed.

Ampuero, A. Zapata (2015). Sostenibilidad y Rentabilidad de las empresas. Perú - Lima: Esam ediciones

Bartra Montilla, J., \& Gonzáles Rivera, J. L. (2019). El sistema de inventarios y su relación la sostenibilidad económica y financiera de la empresa "Fumigaciones Gaviria SAC”. Universidad Nacional de San Martín. http://repositorio.unsm.edu.pe/handle/11458/3271

Bravo, J. Carrasco (2009). Compras e Inventario. España: Ediciones Diaz De Santos, S.A.

Concytec (2020, p.5), La Superintendencia Nacional de Educación Universitaria, a través del Consejo de ciencia, tecnología y comunicación. http://www.untels.edu.pe/documentos/2020_09/2020.09.22_formuacionproyecto s.pdf

Córdova I (2013), En su libro de estadística básica aplicada, ed. 1R - San Marco EIRLtda. ISBN. 978 -612-302-805-3.

Cruz, A. (2017). Gestión de Inventarios (Primera ed.). Málaga: IC Editorial.

Calderon, J. (2008), Estados financieros: Formulación, análisis e interpretación, Segunda ed. JCM. Editores.

Coello, C. (2017). Finanzas Corporativas: Valor llave para una organización sana y competente. IMCP.

Díaz, D. (2017). El control de inventario y sus incidencias en la rentabilidad de las micro y pequeñas empresas del sector de imprentas.

http://repositorio.uladech.edu.pe/bitstream/handle/123456789/4436/CONTR

OL_DE_INVENTARIO_DIAZ_CHIRINOS_DANNY_JACKELINE.pdf?sequ ence $=1 \&$ isAllowed $=\mathrm{y}$

Douglas T. (2000), Control de Inventarios. México: Prentice Hall. 2000

Escribano, M. \& Jiménez, A. (2014). Análisis contable y financiero. Bogotá, ediciones de la U.

Ferrer, A. (2014), Formulación, análisis e interpretación de estados financieros. primera ed. Editorial Ferrer EIRL. 
Ferrer, J. Cárdenas (2014) Gestión del cambio. LID Editorial Empresarial: Colombia. https://www.lideditorial.com/libros/gestion-del-cambio

Garrido, I. \& Cejas, M. (2017), Inventory management as a strategic factor in business administration. Maracaibo. Vol. 13, núm. 37, 2017, pp. 109-129

Guevara, M. (2020). Gestión de inventarios. UF0476. Tutor Formación.

https://www.casadellibro.com/libro-uf0476-gestion-de-

inventarios/9788417943523/11405029

Golas, Z. (2020). The effect of inventory management on profitability: Evidence from the Polish food industry: Case study. Agricultural Economics / Zemedelska Ekonomika, 66(5), 234-242. https://doi.org/10.17221/370/2019-AGRICECON

Guzmán, C. A. (2010). Ratios financieros. Madrid: Editorial coordinación general.

Hernández S. (2014) en su libro de Metodología de la Investigación científica, Mc Graw Hill. Santa Fe.

Hillier F. y Lieberman G. (2010). Introducción a la investigación de operaciones. Novena edición. McGRAW-HILL.785p. Cap. 13

INEGI, (2019, p.29). Instituto Nacional de estadística y Geografía. Mexico. ISBN 978607-530-082-5.

https://www.inegi.org.mx/contenido/productos/prod_serv/contenidos/espanol/bv inegi/productos/nueva_estruc/702825189990.pdf.

INEGI, (2019, p.45). Instituto Nacional de estadística y Geografía. Mexico. ISBN 978607-530-082-5.

https://www.inegi.org.mx/contenido/productos/prod_serv/contenidos/espanol/bv inegi/productos/nueva_estruc/702825189990.pdf

Lizcano j. Álvares y Castello E. (2004), Rentabilidad empresarial: Propuesta practica de análisis y evaluación. Editorial. Print A. Porter Comunicación S.L. https://issuu.com/victore.cardozodelgado/docs/rentabilidad_empresarial_propues ta

Medina, O. (2021), Modelo de gestión de inventario y la rentabilidad de la empresa corporación Global INK S.A.C., (Tesis de maestría). Universidad Cesar Vallejo. https://hdl.handle.net/20.500.12692/69046 
Moreira Rosales, L., García Arias, N., Granda Sanmartín, M., Samaniego Salcan, H., \& Lema Espinoza, M. de J. (2019). Impact of the application of an accounting process and inventory management for the DETEICELI company. 6(3), 1-16.

Muller, M. (2008). Fundamentos de administración de inventarios. Colombia: Editorial norma S.A

Meana, P. Coalla (2017). Gestión de Inventarios. España: Ediciones Nobel S.A

Nicuesa, Maite (2013). Recursos tangibles e intangibles en la empresa. Empresariados. http://empresariados.com/recursos tangibles-e-intangibles-en-laempres/ Alarcon Reynaldo 1991

Prempeh, K. (2017). The impact of efficient inventory management on profitability: Evidence from selected manufacturing firms in Ghana. MPRA Paper.

https://www.researchgate.net/publication/284023064_The_impact_of_efficient_invento ry_management_on_profitability_evidence_from_selected_manufacturing_firms _in_Ghana

Román, C. L. (2012). Fundamentos de análisis financiero. México: Primera edición

Salas, K. Maiguel, H. \& Acevedo, J. (2017). Inventory Management Methodology to determine the levels of integration and collaboration in supply chain. (artículo científico), Vol. 25 - N²Scielo, 328 - 333. http://dx.doi.org/10.4067/S071833052017000200326

Sekeroglu, G. \& M. Altan. (2017). The Relationship Between Inventory Management And Profitability: A Comparative Research On Turkish Firms Operated In Weaving Industry, Eatables Industry, Wholesale And Retail Industry. https://doi.org/10.5281/zenodo.1093139

Suarez C. (2012), The competencies of teachers in ict: basic structure. Universidad de Valencia - España. vol. 16, núm. 1, 2013, pp. 39-61 - ISSN: 1139-613X. https://www.redalyc.org/pdf/706/70625886003.pdf

Swenseth, S. R., \& Olson, D. L. (2016). Trade-offs in lean vs. Outsourced supply chains. 54(13), 4065-4080.

Vara A. Horna (2012) Desde la idea hasta la sustentación: 7 pasos para elaborar una tesis, 2da ed. Universidad San Martín de Porres.

https://www.administracion.usmp.edu.pe/investigacion/files/7-PASOS-PARA-UNATESIS-EXITOSA-Desde-la-idea-inicial-hasta-la-sustentaci\%C3\%B3n.pdf 
Vizcarra Moscoso, J. E. (2010). Auditoría Financiera: Riesgos, control interno, gobierno corporativo y normas de información financiera. Pacíficos Editores

Ülker,Yakup, Arslan,Öznur, \& Esen,Ömer. (2020). Finansal oranlar aracılı̆̆ıyla stok yönetimi ve karlılık ilişkisinin incelenmesi: Imalat sektöründe bir uygulama / Investigation of the relationship between stock management and profitability by using financial ratios: Üniversitesi Sosyal Bilimler Dergisi, 8(4), 1153-1163. https://doi.org/10.18506/anemon.668756

Winston, W. (2004). Investigación de operaciones: aplicaciones y algoritmos. Cuarta edición. International Thomson Editores, S.A. Capítulos 15 y 16. file://C:/Users/HP/Downloads/Investigacion_de_operaciones_cuarta_edic.pdf 